\title{
CARACTERIZAÇÃO FÍSICA DO LEITO DE SEMENTES DE CEVADA BRASILEIRA EM FUNÇÃO DE SUA UMIDADE
}

\author{
G. R. CARVALHO ${ }^{*}$, T. C. POLACHINI ${ }^{1}$, J. F. LOPES FILHO ${ }^{1}$, J. TELIS-ROMERO ${ }^{1}$ \\ ${ }^{1}$ Universidade Estadual Paulista, Departamento de Engenharia e Tecnologia de Alimentos \\ *e-mail: gisandro_carvalho@ hotmail.com
}

\begin{abstract}
RESUMO
A cevada (Hordeum vulgare L.) está entre as maiores culturas de grãos no mundo, grande parte da produção é transformada em malte, importante matéria prima para a fabricação de bebidas muito populares como a cerveja. Para a produção do malte, o grão de cevada precisa ter a sua umidade alterada e controlada para garantir que todas as transformações ocorram. As alterações na umidade do grão provocam mudanças nas propriedades físicas do mesmo e, consequentemente, do leito partículas. Logo, o conhecimento das propriedades físicas do leito em uma ampla faixa de umidade dos grãos se faz necessária para o correto projeto de operações e equipamentos envolvidos no processamento da cevada. $\mathrm{O}$ aumento da umidade fez com que o peso e o volume do leito se tornassem maiores, assim como a porosidade do mesmo. A densidade real e a densidade bulk diminuíram com a elevação da umidade do grão, assim como a área específica do leito.
\end{abstract}

\section{INTRODUÇÃO}

A cevada (Hordeum vulgare L.) é uma das maiores e mais antigas culturas de grão no mundo, onde a Alemanha, França, Ucrânia e Rússia são os maiores produtores e exportadores (FAO, 2012). No Brasil, a produção de cevada vem aumentando a cada ano.

Grande parte da produção de cevada é destinada à maltagem devido à grande lucratividade deste produto. O malte é uma importante matéria prima da cerveja, que segundo a FAO (2012), teve um aumento de 54\% na produção no período de 1999-2009, e também do whisky, uma das bebidas mais populares do mundo.

A maltagem da cevada garante mudanças físicas e químicas no grão. Três etapas são necessárias para garantir que estas mudanças ocorram. Primeiramente, a cevada é alternadamente imersa em água e seca durante 40 horas até atingir em torno $40 \%$ de umidade. Após esta primeira etapa, os grãos são mantidos sob alta umidade e temperatura por 4-6 dias para germinação, na qual ocorre o crescimento do embrião, síntese de enzimas e limitada deterioração do endosperma. E, finalmente, a cevada germinada é desidratada para garantir sua estabilidade (SOLOGUBIK et al., 2013).

O conhecimento da morfologia e das dimensões dos grãos é essencial para o projeto de equipamentos de limpeza, classificação e separação (KACHRU, GGUPTA \& ALAM, 1994). Assim como as dimensões características permitem o cálculo de parâmetros necessários para o projeto da secagem e aeração (AL-MAHASNEH \& RABABAH, 2007).

A densidade bulk é utilizada para determinar a capacidade de armazenamento e 
transporte, enquanto a densidade real é útil para o projeto de equipamentos de separação. Além disso, a porosidade é utilizada para determinar a resistência a passagem do ar durante a secagem ou a aeração (BROOKER, BAKKER-ARKEMA \& HALL, 1992; KACHRU, GGUPTA \& ALAM, 1994).

$\mathrm{Na}$ literatura, há poucas informações sobre as propriedades físicas de grãos de cevada em uma ampla faixa de umidade, normalmente utilizada no processo de maltagem. Portanto, devido à necessidade destas informações, este trabalho teve como objetivo determinar algumas propriedades do leito de grão de cevada variedade Scarlett em uma faixa de umidade de 12,44 a $45,31 \%$, em base seca.

\section{MATERIAL E MÉTODOS}

A cevada utilizada neste trabalho foi da variedade Scarlett, produzida na região sul do Brasil, no estado do Paraná. Para utilização, foi realizada uma limpeza manual dos grãos para remoção de sujidades, corpos estranhos e sementes imaturas.

$O$ teor de umidade dos grãos foi determinado, em triplicata, pela diferença de peso medida em uma balança analítica (modelo AUX220, Shimadzu, Japão) após aproximadamente 24 horas em estufa a 105 ${ }^{\circ} \mathrm{C}$. A umidade inicial dos grãos foi de $12,44 \%$, em base seca.

A partir da umidade inicial, os grãos foram umidificados adicionando água destilada com auxílio de um borrifador, para obtenção de novas umidades. Feito isso, os grãos foram acondicionados em sacos plásticos impermeáveis ao vapor d'água e mantidas no refrigerador a $5 \pm 1{ }^{\circ} \mathrm{C}$ por seis dias, para permitir uniformidade na umidade de toda a amostra. Antes de cada medida, as amostras eram retiradas do refrigerador com algumas horas de antecedência para se ambientarem com a temperatura do laboratório.
Figura 1 - Aparato utilizado para a medida de volume.
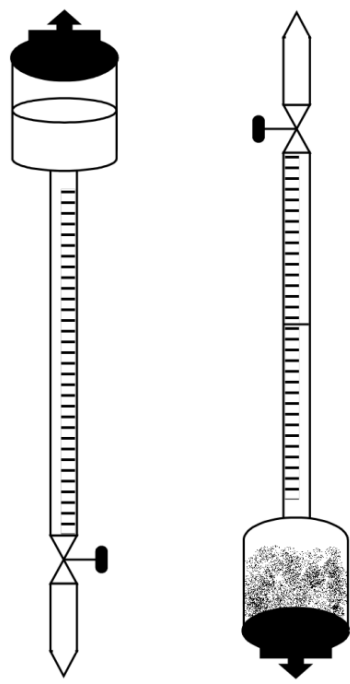

Fonte: GABAS et al., 2005.

As umidades foram determinadas a partir da Equação (1):

$$
M_{a}=\frac{M_{i}\left(X_{f}-X_{i}\right)}{X_{i}+100}
$$

Todas as propriedades físicas do leito de cevada foram determinadas na faixa de umidade obtida $(12,44-45,31 \%$, em base seca). Para cada parâmetro foram realizadas 10 replicatas em cada valor de umidade.

O peso de 1000 grãos $\left(P_{1000}\right)$ foi obtido através de 100 grãos selecionados aleatoriamente. $\mathrm{O}$ peso deles foi medido em uma balança analítica (modelo AUX220, Shimadzu, Japão), com precisão de 0,0001 g, e então multiplicado por 10 , resultando no peso de 1000 grãos. Também foram realizadas 10 replicatas para cada valor de umidade (COSKUNER \& KARABABA, 2007; SOLOGUBIK et al., 2013).

Através do sistema mostrado na Figura 1 e descrito por Gabas et al. (2005), foi determinado o volume de 1000 sementes de cevada em cada umidade através do deslocamento de tolueno. 
Com os valores de peso e de volume dos 1000 grãos, obtém-se então a densidade real do leito, através da relação:

$$
\rho_{r}=\frac{m_{P}}{v_{P}}
$$

A densidade bulk foi determinada através da relação entre o peso de uma amostra de grãos e o volume total que a mesma ocupa (Equação 3). O peso dos grãos foi obtido com auxílio de uma balança analítica (modelo AUX220, Shimadzu, Japão), com precisão de 0,0001, para um volume total de $250 \mathrm{~mL}$ (DESHPANDE, BAL \& OJHA, 1993; VILCHE, GELY \& SANTALLA, 2003).

$$
\rho_{b}=\frac{m_{P}}{v_{T}}
$$

A porosidade do leito é definida como a porcentagem do leito que não é ocupada pelos grãos. Essa porcentagem é calculada pela seguinte equação (MOHSENIN, 1986):

$$
\varepsilon=1-\frac{\rho_{b}}{\rho_{r}} \times 100
$$

A área específica do leito foi obtida através da relação entre a área específica da partícula e a porosidade do leito (Equação 5).

$$
S_{L}=S_{P}(1-\varepsilon)
$$

Os resultados obtidos para as propriedades físicas de grãos foram submetidos à análise de variância (ANOVA) e regressão com auxílio do software Microsoft EXCEL ${ }^{\circledR}$.

\section{RESULTADOS E DISCUSSÃO}

\subsection{Peso e Volume do Leito}

O peso de 1000 grãos aumentou $(p<0,001)$ linearmente de 43,43 para $56,97 \mathrm{~g}$ com a elevação da umidade de 12,44 para $45,31 \%$ (b.s.), assim como o volume de 1000 grãos, que variou de 35,58 a $48,96 \mathrm{~cm}^{3}$ (Figura 2). $\mathrm{O}$ desvio padrão foi menor que $0,56 \mathrm{~g}$ em todos os casos. As relações entre o peso e o volume de 1000 grãos e a umidade são dadas por:

$P_{1000}=38,184+0,4135 X_{c}$
$V_{1000}=30,306+0,4076 X_{c}$

com valores de $R^{2}$ de 0,9996 e 0,999, respectivamente..

Resultados semelhantes foram obtidos por Sologubik et al. (2013) ao avaliar algumas propriedades físicas de cevada da variedade Scarlett produzida na argentina e em estudos com trigo verde, soja, grão de bico e sementes de cominho (ALMAHASNEH \& RABABAH, 2007; DESHPANDE, BAL \& OJHA, 1993; DUTTA, NEMA \& BHARDWAJ 1988; SINGH AND GOSWAMI, 1996).

Figura 2 - Variação do (ロ) peso e do ( $\square$ ) volume de 1000 grãos de cevada em função da umidade e dados para peso de 1000 grão por SOLOGUBIK et al. (2013) (-).

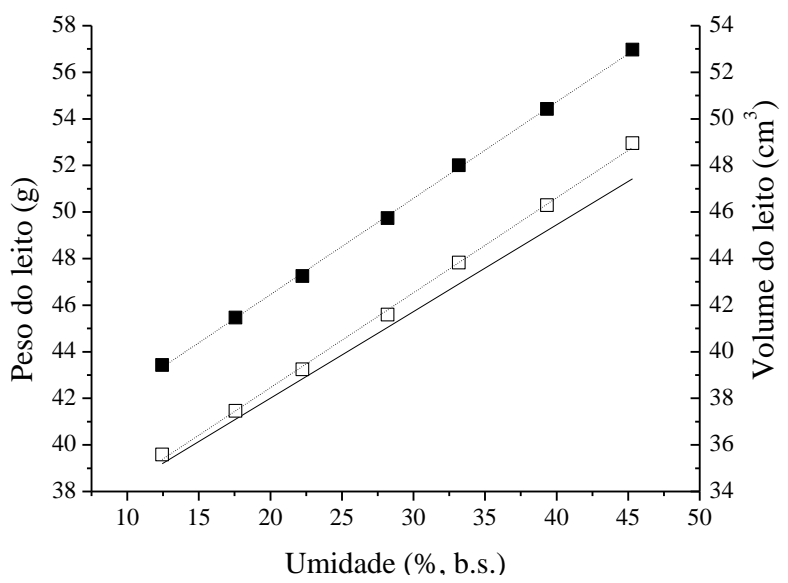


Figura 3 - Variação da (ロ) densidade real do leito e da ( $\square$ ) densidade bulk de grãos de cevada em função da umidade e dados para densidade real e bulk por SOLOGUBIK et al. (2013) (-).

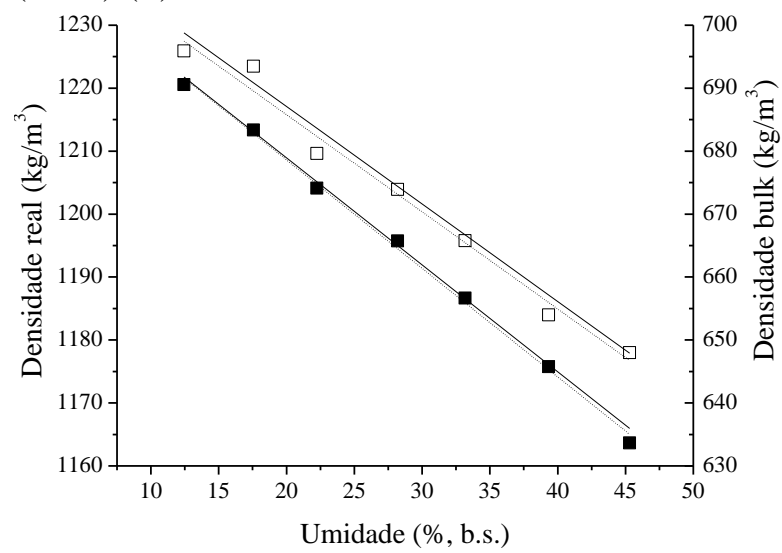

\subsection{Densidade Bulk do Leito}

A densidade bulk $\left(\rho_{b}\right)$ do leito de grãos de cevada diminuiu significativamente $(p<0,01)$ de 695,89 para $647,99 \mathrm{~kg} / \mathrm{m}^{3}$ com o aumento da umidade de 12,44 para $45,31 \%$ (b.s.) (Figura 3). As médias apresentaram desvio padrão de no máximo $9,96 \mathrm{~kg} / \mathrm{m}^{3}$. Esse comportamento pode ser atribuído ao fato de que o aumento no peso da amostra associado ao aumento da umidade foi menor que a expansão sofrida pelo grão, ou seja, o volume de ar entre os grãos mais úmidos é menor que o volume de ar entre os grãos mais secos.

Este fenômeno causaria uma maior compactação (maior densidade bulk) nos grãos mais secos quando comparados com os grãos mais úmidos. A relação entre a densidade bulk $\left(\rho_{b}\right)$ e a umidade é dada por:

$\rho_{b}=716,64-1,5429 X_{c}$

$\operatorname{com} R^{2}=0,9846$.

Sologubik et al. (2013) obtiveram valores muito próximos para densidade bulk de cevada da variedade Scalett argentina. Corrêa et al. (2013) avaliaram características físicas de três variedades de cevada: brasileira: Brau, Caue e Elis, obtendo valores de 750,730 e $740 \mathrm{~kg} / \mathrm{m}^{3}$, respectivamente, para uma umidade de aproximadamente $7 \%$ (b.s.), valores estes, que estão próximos, mas um pouco maiores que os encontrados neste trabalho. Resultados semelhantes também foram obtidos no estudo de outros grãos (DESHPANDE, BAL \& OJHA, 1993; DUTTA, NEMA \& BHARDWAJ 1988).

\subsection{Densidade Real do Leito}

A densidade real $\left(\rho_{r}\right)$ dos grãos de cevada diminuiu significativamente $(p<0,01)$ de 1220,56 para $1163,65 \mathrm{~kg} / \mathrm{m}^{3}$, com o aumento da umidade dos grãos (Figura 1Figura 3). Esta redução na densidade real se dá, pois, o aumento no volume dos grãos é maior que o aumento no peso provocado devido à adição de água. Também pode ser explicado pelo fato da água ter uma densidade menor que a do grão.

A relação entre a densidade real e a umidade dos grãos é dada por:

$\rho_{r}=1243-1,7225 X_{c}$

$\operatorname{com} R^{2}=0,9976$.

Dados bem próximos de densidade real de cevada argentina da mesma variedade foram reportados por Sologubik et al. (2013). Corrêa et al. (2013) obtiveram valores de $1280 \mathrm{~kg} / \mathrm{m}^{3}$ para cevada das variedades Brau e Elis e $1270 \mathrm{~kg} / \mathrm{m}^{3}$ para variedade Caue. Outros grãos apresentaram resultados similares (DESHPANDE, BAL \& OJHA, 1993)

\subsection{Porosidade do Leito}

A porosidade $(\varepsilon)$ foi calculada pela Equação 4, em função da densidade real e densidade bulk. Pode ser observado que a porosidade aumentou significativamente $(p<0,01)$ de 42,99 para $44,31 \%$ com o aumento da umidade, como mostrado na Figura 4. 
Figura 4 - Efeito da umidade na (ש) porosidade do leito de cevada e dados por SOLOGUBIK et al. (2013) (-).

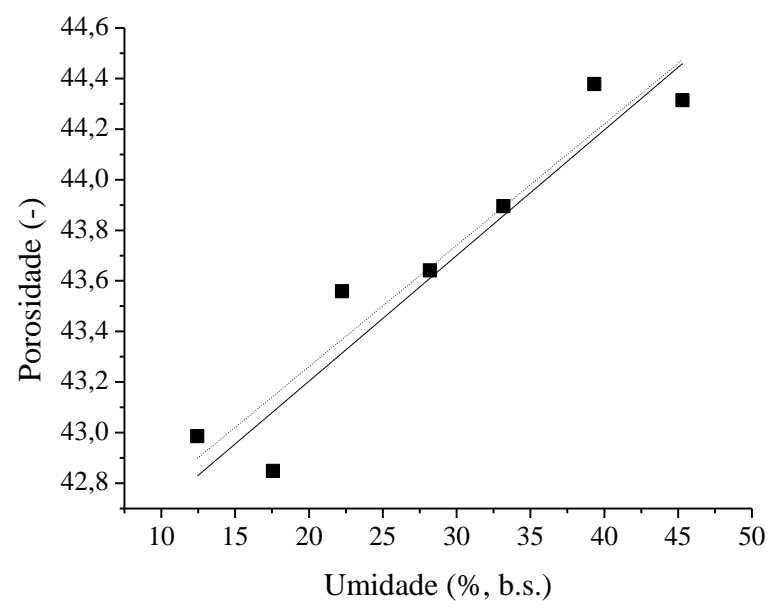

O motivo desse aumento pode ser o seguinte: enquanto os grãos absorvem umidade, seus volumes individuais aumentam, consequentemente suas formas mudam e também o volume do leito. Este comportamento provoca a redução do número de grãos ocupando um volume fixo, e então, a densidade bulk diminui. Em outras palavras, para uma dada massa de grãos, um aumento de sua umidade resulta em um maior volume do leito. Analogamente, a adição de água na estrutura do grão resulta no aumento de sua densidade real, mas a mudança causada no volume devido a umidificação influencia mais na densidade bulk do que na densidade real do grão, aumentando então a porosidade (SOLOMON \& ZEWDU, 2009).

A relação entre a porosidade $(\varepsilon)$ e a umidade é dada por:

$\varepsilon=42,302+0,048 X_{c}$

$\operatorname{com} R^{2}=0,9089$.

Comportamento similar foi obtido por Sologubik et al. (2013) ao estudarem sementes de cevada argentina da variedade Scarlett e por Solomon \& Zewdu (2009) ao estudarem niger seed. A porosidade de cevada brasileira das variedades Brau, Caue e Elis, apresentadas por Corrêa et al. (2013) foram próximas às obtidas neste trabalho.

\section{5 Área Específica do Leito}

A área especifica do leito, obtida a partir da Equação 5, apresentou uma redução significativa $(p<0,001)$ de 8,383 até $7,361 \mathrm{~cm}^{-}$ 1 com o aumento da umidade de 12,44 até $45,31 \%$ (b.s.) (Figura 5). O maior valor de desvio padrão foi de $0,014 \mathrm{~cm}^{-1}$.

A relação entre a área específica do leito e a umidade é dada pela seguinte equação:

$$
S_{L}=8,7812-0,0322 X_{c}
$$

$\operatorname{com} R^{2}=0,9938$.

A redução da área específica do leito se dá devido ao aumento no volume das partículas provocado pelo aumento da umidade, já que a área específica é definida pela área superficial total dos grãos que preenchem um volume fixo de leito. Esse aumento no volume das partículas reduz a quantidade de partículas que preenchem um determinado volume de leito, acompanhado por um menor empacotamento do mesmo e consequente aumento na porosidade. Todos estes fenômenos contribuem para a redução da área específica do leito.

Figura 5 - Efeito da umidade na área específica (ロ) do leito de cevada.

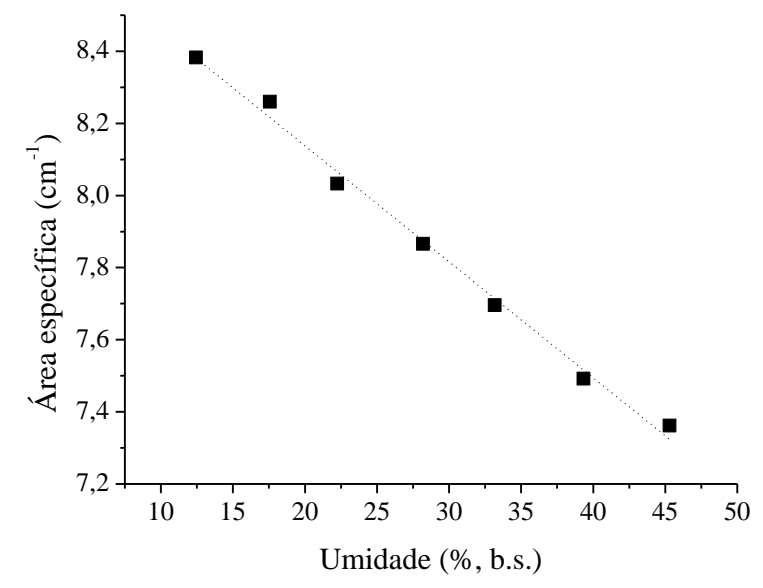




\section{CONCLUSÃO}

Como foi mostrado no trabalho, as propriedades do leito sofreram alterações significativas para a umidade dos grãos variando de 12,44 a $45,31 \%$ (b.s.).

O peso de $1000\left(P_{1000}\right)$ grãos aumentou linearmente $\left(R^{2}=0,9996\right)$ com a elevação da umidade, variando de 43,43 até 56,97 g.

As densidades real e bulk reduziram linearmente $\left(R^{2}>0,984\right)$ com o aumento da umidade, com valores de 1220,56 a 1163,65 $\mathrm{kg} / \mathrm{m}^{3}$ e de 695,89 a $647,99 \mathrm{~kg} / \mathrm{m}^{3}$, respectivamente.

O acréscimo na umidade do grão provocou um aumento na porosidade do leito $(\varepsilon)$, enquanto que a área específica $\left(S_{L}\right)$ do mesmo reduziu, variando de 42,99 para $44,31 \%$ e de 8,383 para 7,361 $\mathrm{cm}^{-1}$, respectivamente.

A elevação da umidade resultou em uma expansão do leito, aumentando o volume das partículas e consequentemente do leito, aumento esse que foi mais intenso que o ganho de peso da partícula, além de promover um menor empacotamento, tonando a porosidade maior e reduzindo a área específica e as densidades real e bulk.

\section{NOMENCLATURA}

$M_{a} \quad$ Massa de água adicionada na $\mathrm{kg}$ amostra

$M_{i} \quad$ Massa inicial da amostra $\quad \mathrm{kg}$

$m_{p} \quad$ Peso das partículas $\quad \mathrm{kg}$

$p \quad$ Coeficiente de correlação de (-) Pearson

$P_{1000}$ Peso de 1000 partículas $\quad \mathrm{kg}$

$R^{2} \quad$ Coeficiente de correlação $\quad(-)$

$S_{L} \quad$ Área específica do leito $\quad \mathrm{cm}^{-1}$

$S_{p} \quad$ Área específica da partícula $\quad \mathrm{cm}^{-1}$

$v_{p} \quad$ Volume das partículas $\mathrm{m}^{3}$

$v_{T} \quad$ Volume do leito $\mathrm{m}^{3}$

$V_{1000}$ Volume de 1000 partículas $\mathrm{cm}^{3}$

$X_{c} \quad$ Umidade da amostra $\%$, b.s.

$X_{f} \quad$ Umidade final da amostra $\%$, b.s.
$X_{i} \quad$ Umidade inicial da amostra

$\%$, b.s.

$\varepsilon \quad$ Porosidade do leito

$(-)$

$\rho_{b} \quad$ Densidade bulk

$\rho_{r} \quad$ Densidade real do leito

$\mathrm{kg} / \mathrm{m}^{3}$

$\mathrm{kg} / \mathrm{m}^{3}$

\section{REFERÊNCIAS}

AL-MAHASNEH, M.A., RABABAH, T.M. Effect of moisture content on some physical properties of green wheat. J. Food Eng. v.79, p.1467-1473, 2007.

BROOKER, D.B., BAKKER-ARKEMA, F., Hall, C.W. Drying and Storage of Grains and Oilseeds. Van Nostrand Reinold, NY. 1992.

CORRÊA, C., GOUVÊA, L. F. C., FREIRE, J. T., MAIA, G. D. Caracterização física e fisiológica de sementes de cevada brasileira para fins de produção de malte cervejeiro. Anais do XXXVI Congresso Brasileiro de Sistemas Particulados. v. 1, p. 106-115, Maceió, AL, 2013.

COSKUNER, Y., KARABABA, E. Some physical properties of flaxseed (Linum usitatissimum L.). J. Food Eng. v.78, p.1067-1073, 2007.

DESHPANDE, S.D., BAL, S., OJHA, T.P. Physical properties of soybean. J. Agric. Eng. Res. v.56, p.89-98, 1993.

DUTTA, S.K., NEMA, V.K., BHARDWAJ, R.K. Physical properties of gram. J. Agric. Eng. Res. v.39, p.259-268, 1988.

FAO, 2012. FAOSTAT. Food and Agriculture Organization of the United Nations. Disponível em: http://faostat.fao.org/faostat. Acesso em 10 jun 2015. 
GABAS, A. L.; MARRA-JÚNIOR, W. D.; TELIS-ROMERO, J.; TELIS, V. R. N. Changes of Density, Thermal Conductivity, Thermal Diffusivity, and Specific Heat of Plums During Drying. International Journal of Food Properties, v.8, n.2, p.233-242, 2005.

KACHRU, R.P., GUPTA, R.K., ALAM, A. Physico-chemical constituents and engineeringproperties of food crops, 1st ed. Scientific Publishers, Jodhpur, India. 1994.

MOHSENIN, N. N. Physical Properties of Plant and Animal Materials. Gordon and Breach Science Publishers, NY, USA. 1986.

SOLOGUBIK, C.A.; CAMPAÑONE, L. A.; PAGANO, A. M.; GELY, M. C. Effect of moisture content on some physical properties of barley. Industrial Crops and Products, v.43, p.762-767, 2013.

SOLOMON, W.K., ZEWDU, A.D. Moisturedependent physical properties of niger (Guizotia abyssinica Cass.) seed. Ind. Crops Prod. v.29, p.165-170, 2009.

VILCHE, C., GELY, M., SANTALLA, E. Physical properties of quinoa seeds. Biosyst.Eng. v.86(1), p.59-65, 2003 